RAD Conference Proceedings, vol. 2, pp. 167-172, 2017

www.rad-proceedings.org

now0001 tht

\title{
SPECIFIC APPROACHES TO PLANNING OF PATIENTS WITH PRIMARY AND SECONDARY BRAIN TUMORS USING THE TOMOTHERAPY PLANNING SYSTEM
}

\author{
S.P. Odarchenko, M.B. Gumeniuk* ${ }^{*}$ K.V. Gumeniuk, O.V. Zinvaliuk, D.S. Sinchuk \\ LLC “Ukrainian Center of Tomotherapy”, Kropyvnytskyy, Ukraine
}

\begin{abstract}
Nowadays only several medical centers of Ukraine are equipped with special linear accelerators for treating patients using Image-Guided Radiation Therapy (IGRT) and Intensity-Modulated Radiotherapy (IMRT), apart from 3DCRT methods. Sequential (SEQ) and Simultaneous Integrated Boost (SIB) are common IMRT techniques for whole brain irradiation and metastasis treatment using TomoTherapy system TomoHD. The radiotherapy system, mentioned above, is innovation for oncological diseases treatment in Ukraine as well as in post Soviet Union countries in general. Thus, one of main goals of the work is to define the quality of different therapeutic plans, to identify and describe the main features of treatment plans preparation and creating and to describe some specific approaches for SIB and SEQ techniques in planning of patients with brain tumors using the TomoTherapy Planning System.
\end{abstract}

Key words: Brain cancer, conformity index, homogeneity index, IGRT, IMRT, sequential and simultaneous integrated boost, TomoTherapy Tomo HD

DOI: $10.21175 /$ RadProc.2017.34

\section{INTRODUCTION}

The Intensity-Modulated Radiotherapy (IMRT) is the most suitable radiotherapy technique for brain treating in Ukrainian center of TomoTherapy. It is provided in two ways: Sequential (SEQ) and Simultaneous Integrated Boost (SIB). The purpose of this work is to define the quality of therapeutic plans in different cases, to identify and describe the main features of treatment plans creating and to describe some specific approaches for SIB and SEQ techniques in planning preparing of patients with brain tumors using the TomoTherapy Planning System.

For the SIB technique, the different dose is delivered to PTV-LR and PTV-HR simultaneously in one fraction, during the same procedure. The number of fractions of SIB is less than SEQ.

For SEQ technique, the planning target volume-low risk (PTV-LR) is irradiated in the first plan and after the end of first course; the dose is boosted to the planning target volume-high risk (PTV-HR) with the second plan in the second course. Both plans have the same dose per fraction during the whole treatment.

The patients are treated with the radiotherapy system TomoTherapy Tomo HD.

\section{MATERIALS AND METHODS}

\subsection{Contouring}

25 therapeutic plans, which were used for treating patients with brain tumors, were analyzed. They were created in the time range from June 2015 till the end of 2016. All patients were treated by using the radiotherapy system TomoTherapy Tomo HD.

The contours of the target (GTV, CTV, PTV (CTV + $0.3 \mathrm{~cm})$ ), and critical organs (eyes, lenses, optical nerves, chiasm, the brain and brainstem) were determined by radiation therapists with using MiM Maestro contouring software. The PTV1 for SIB included GTV for metastases which were defined as the contrast-enhancing lesion on T1-weighted MRI plus 3 $\mathrm{mm}$ uniform margin. The PTV2 for WBRT included the whole brain plus $3 \mathrm{~mm}$ margin excluding PTV1. After the end of therapists contouring, medical physicists created different additional "logical" structures. The structures are unique for different plans and techniques and are used for a better control of dose distribution in the target and around it, especially in the critical volumes and areas.

\subsection{Dividing structures}

If the organ at risk (OAR) has common parts with the target (overlapped structures), it is important to avoid the dose getting maximums in the common areas

*gymenick@gmail.com 
of the target and OAR. For that reason, OAR is divided into substructures (Fig. 1):

PTV OAR is the part of OAR volume, which is fully located in the PTV. In this volume it is very important to control the maximum dose.

OAR plan (OAR-) is part of OAR, which is located out of the PTV volume.

In this volume a high dose gradient for maximum protection of the part of OAR must be reached.

The part of PTV, which does not contain any common volume with OARs, is called as PTV plan.

So we get the situation, where PTV consists of smaller parts with a different dose coverage priority, such as PTV OAR (one or more) and OAR plan.

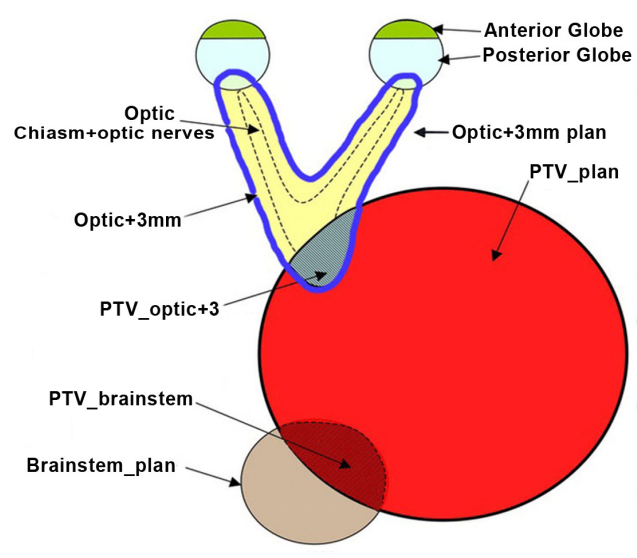

Figure 1. Schematic results of dividing volumes into "logical" sub-volumes for planning

The real dose distribution is controlled and evaluated by using Dose Volume Histogram (DVH) curves, which corresponds to the real OARs and PTVs.

\subsection{Dmin structures}

Dmin1 is an additional circular (spherical) structure, created around a target.

Dmin2 is the second additional circular (spherical) structure created around Dmin1 (Fig. 2).

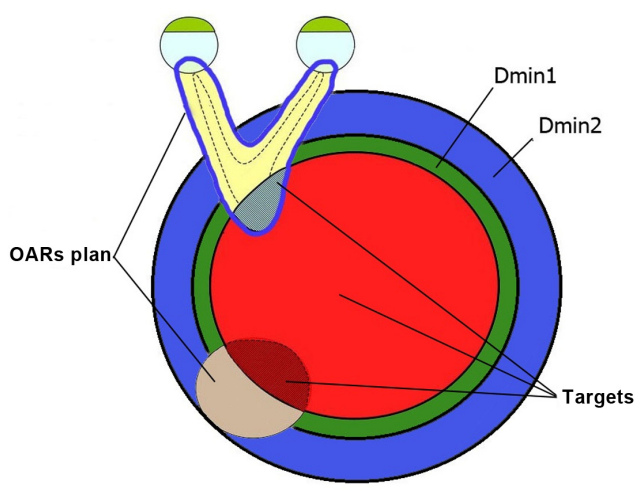

Figure 2. Schematic view of Dmin1 and Dmin2 creating
Their main goals are:

- to ensure good conformity and steep gradient of high doses around the target;

- to give additional control over the maximum dose beyond the target volume;

- to ensure uniformity and decline dose in regions of medium and low doses outside the target volume.

Dmin1 is $5 \mathrm{~mm}$ outer ring and Dmin2 is second ring with size of 20-25 $\mathrm{mm}$ are shown.

\subsection{Simultaneous integrated boost (SIB) structures}

If it is necessary to irradiate overlapped targets with different doses, one or two ring structures RingX are made, which provide a uniform transition of the dose from PTV HR to PTV LR (Fig. 3). Also, for nonoverlapping volumes, a special logical volume PTVLR_nR (orange region in Figure 3) is created by extracting previously made rings from PTV LR for a more clear DVH and statistical analysis.

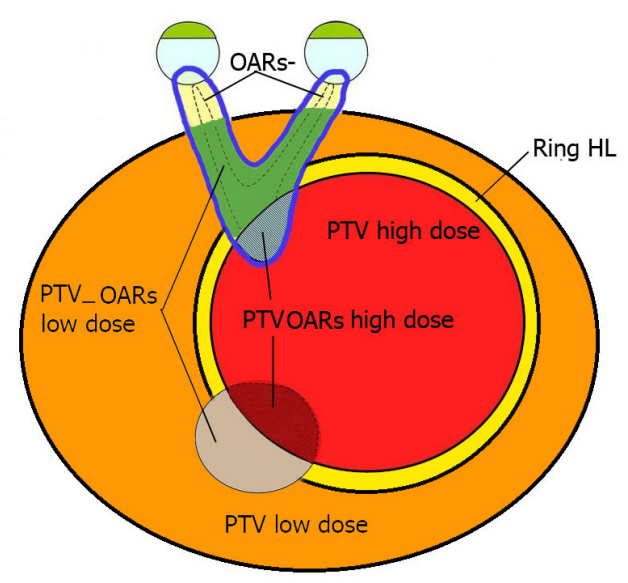

Figure 3. Schematic results of using rings for SIB planning

\subsection{Plan calculation}

After creating all additional structures, CT and RT structures files are sent to the planning station. All dosimetric calculations were made by using TomoTherapy planning system. All structures are divided into two groups: targets and OARs. Each group has its own hierarchy.

Besides, additional parameters must be selected before starting the planning procedure (Modulation factor (MF), Field width (FW), Mode (IMRT or 3D), IVDT curve, red lasers position).

The optimization of the plan is started after calculating the dose beamlets and inserting the necessary parameters and values.

\subsection{Automatic workflows}

Preparation for plan calculating and plan optimizing are very time consuming processes.

Standard TomoTherapy software has poor functionality and there is no possibility of CT and MRI images fusion for example. Also there is no possibility of different "logical" structures creation (no "Boolean 
operations" available). So for delineation of different structures third party contouring software is needed. At UCT department MIM maestro software is used. It is a commercial software solution for radiation oncology.

Reducing of beamlet calculation time is very important, but the only solution of the problem is upgrading of the system to the VoLO (Voxel-Less Optimization) Technology. To make workflow faster we decided to reduce the additional structures creating time. Automatic workflow presets were made in MIM software for main treatment sites. After finishing of targets and OARs delineation by the physician (default list of "empty" structures with default names must be chosen before starting of delineation), medical physicist makes revision of all structures contours and, in most cases, launches automatic workflow preset realization. All additional contours are generated automatically (Fig. 4). This task usually takes 2-5 minutes. In case of manual creation of all logical structures, half an hour or sometimes even more time was needed.

After the end of contours creation CT images and RT structures files are sent to Tomoserver.

In TomoTherapy planning software presets for different sites were also created (Fig. 5). The chosen preset fills automatically all necessary data of plan properties (FW, pitch, modulation factor, etc.) and structures initial priority, importance and dose restrictions (Fig. 6). Beamlet calculation may be started immediately in background mode. Structure properties importance and restrictions may be changed during the optimization, but very often the protocols initial values (experience based values are set) are good enough for getting fine planning results (Fig. 7).
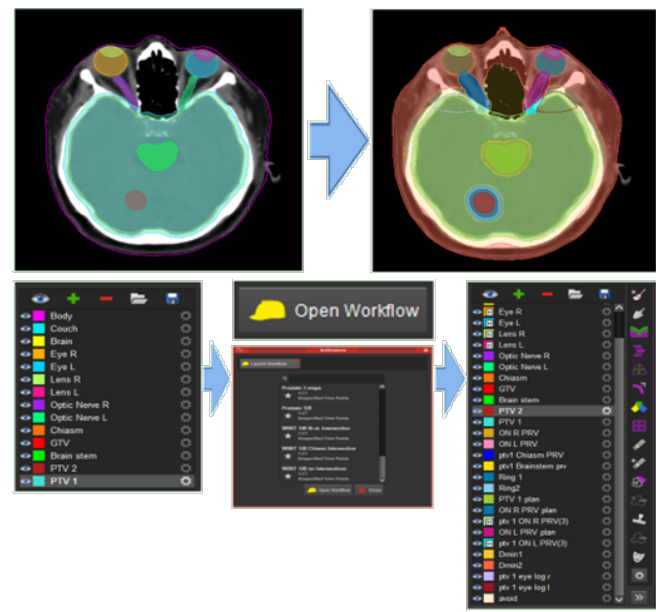

Figure 4. Automatic workflow realization results

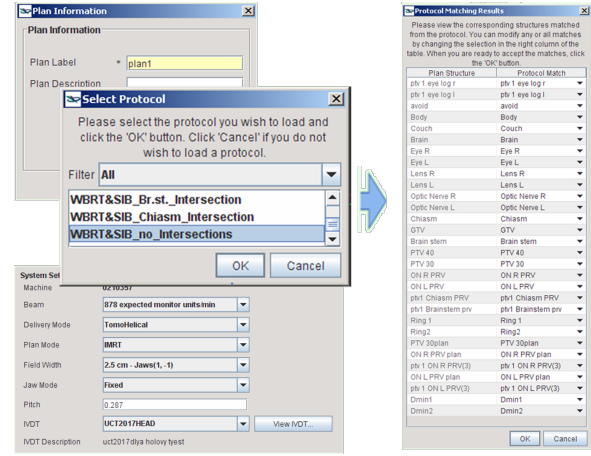

Figure 5. TomoTherapy presets

\subsection{Preliminary assessment plan}

The aim is to obtain such a dose distribution in which $95 \%$ of the prescribed dose covers $99 \%$ of the target. At least, $95 \%$ of the PTV volume must be covered by $95 \%$ of the prescribed dose, if OAR is partially or fully included in PTV, and there is no possibility to irradiate it with the full prescribed dose [5]. Each individual case is always discussed with a therapist.

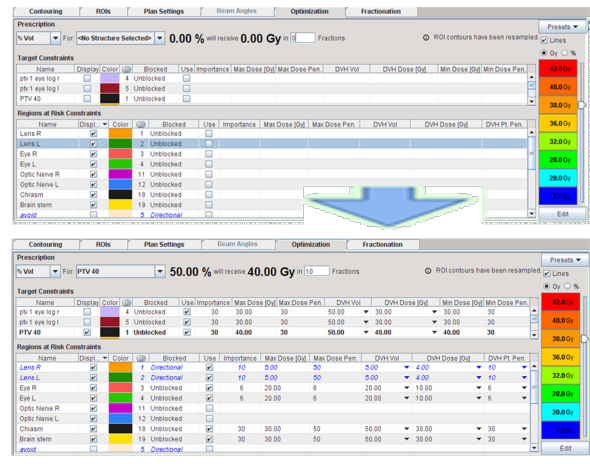

Figure 6. The example result of the presets use

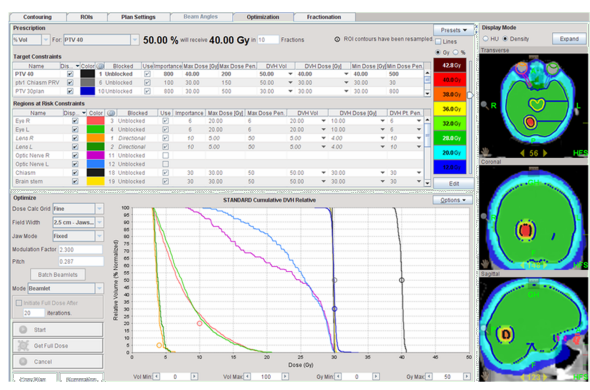

Figure 7. The example of planning results 
The maximum dose in point should not exceed $107 \%$ of the prescribed dose or, in some complicated cases, D1\% should not exceed $107 \%$ of the prescribed dose (Fig. 8).

For dose distribution uniformity analyzing, the dose homogeneity index (HI) is used, which is calculated according to (1).

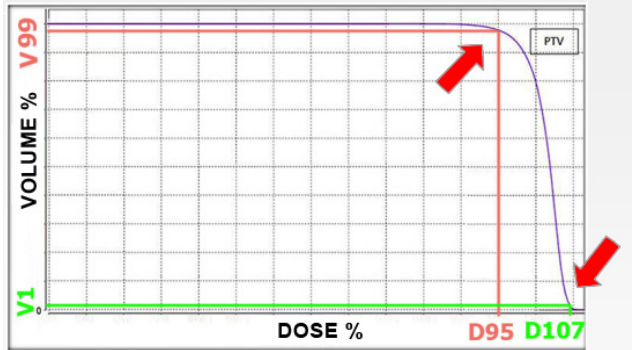

Figure 8. Schematic view of DVH for dose distribution analysis

2.8. Analysis of dose distribution uniformity

$$
H I=\frac{D 1 \%-D 99 \%}{D 50 \%}
$$

where D $1 \%$ is dose of $1 \%$ volume of the PTV (maximum dose), D99\% is dose of $99 \%$ volume the PTV (minimum dose), D50\% is median dose [1].

A value of $\mathrm{O}$ corresponds to absolute dose homogeneity within the PTV.

\subsection{Analysis of dose conformity}

Paddick CI is a conformity index that shows how well the target is covered by the dose (95\% of the prescribed dose) and assesses how close the 95\% isodose line is to the target [2].

CI value can vary between 0 and 1 , the value of 1 is the most accurate target coverage without the reference dose exposure of healthy surrounding tissues, a value of $o$ indicates a lack of conformity which may arise in the case of "miss" or when it is a large amount of irradiated healthy tissue [3].

$$
C I=\left(\frac{T V D r x}{T V}\right) \cdot\left(\frac{T V D r x}{V D r x}\right)
$$

where TV is volume of the target,

TVDrx volume of target receiving 95\% or higher percent of the prescribed dose

VDrx is total volume, receiving $95 \%$ or higher percent of the prescribed dose.

\subsection{Plan verification}

Plans verification was performed with the software PTW VeriSoft and Octavius phantom with detector 2D-array 729 (PTW). Test results were within 97\% - 100\% (analysis of the following parameters: Gamma 3D, 3mm distance to agreement $/ 3 \%$ dose difference with ref. to the local dose).

\section{RESULTS}

Prescribed doses were:

DPTV46=46 and DPTV6o=60 Gy ( $2 \mathrm{~Gy} / \mathrm{fr}$ ) for SEQ IMRT technique per 23 and 30 fractions respectively.

DPTV LR=30 and DPTV HR=40 Gy $(3-4 \mathrm{~Gy} / \mathrm{fr})$ for SIB IMRT technique per 10 fractions.

Medical plans features: field width was $1.0-2.5 \mathrm{~cm}$ pitch was 0.287; initial modulation factor was 3,4 ; real modulation factor range varied from 2.1 to 2.7 . Optimization options were set in the next way: the value of the average dose that covered VPTV 100\% met the prescribed dose, the dose (DPTV 99\%), which covered the VPTV $99 \%$ was greater than $95 \%$ of the prescribed dose, in severe cases the dose that covered VPTV $95 \%$ was greater than $95 \%$ of the prescribed dose. Maximum dose in point was Dmax $\leq 107 \%$ of the prescribed dose.

The minimum dose at point Dmin was $>90 \%$ of the prescribed dose.

The resulting 17 SEQ plans were characterized by the high uniformity of dose distribution DPTV95\% = 97-100\%, Dmax was $102-105 \%$, Dmean was $100 \%$, the average HImean was 0.048, HImax was 0.123 , HImin was 0.0175 , the average value CImean was 0.86 , CImax was 0.994 , CImin was 0.673 . The average exposure time was $328 \mathrm{~s}$. (From $252 \mathrm{~s}$ to $432 \mathrm{~s}$ ) (Table 1).

For 8 SIB plans the results are shown in Table 2.

The resulting 8 SIB plans were characterized by the high uniformity and conformity of dose distribution:

a. for PTV HR:

HImean was 0.066; HImax was 0.113; HImin was 0.051.

CImean was 0.755; CImax was 0. 9; CImin was 0.734 .

b. for PTVLR_nR:

HImean was 0.074; HImax was 0.081; HImin was o.063.

CImean was 0.838; CImax was 0.852; CImin was 0.754 .

The average exposure time Tav was $411 \mathrm{~s}$. (From $384 \mathrm{~s}$ to $450 \mathrm{~s}$ ).

Table 1. CI, HI and time of SEQ plans

\begin{tabular}{|c|c|c|}
\hline T min & HI & CI \\
\hline 4.2 & 0.058 & 0.863 \\
\hline 4.4 & 0.124 & 0.895 \\
\hline 7.2 & 0.059 & 0.884 \\
\hline 5.8 & 0.072 & 0.860 \\
\hline 5.7 & 0.035 & 0.880 \\
\hline 5.8 & 0.033 & 0.870 \\
\hline 5.8 & 0.039 & 0.887 \\
\hline 5.6 & 0.057 & 0.901 \\
\hline 5.5 & 0.022 & 0.672 \\
\hline 5.8 & 0.017 & 0.746 \\
\hline 6.8 & 0.032 & 0.857 \\
\hline 5.0 & 0.037 & 0.857 \\
\hline 5.2 & 0.038 & 0.869 \\
\hline 4.8 & 0.047 & 0.994 \\
\hline 5.8 & 0.053 & 0.880 \\
\hline 4.2 & 0.026 & 0.722 \\
\hline 5.7 & 0.064 & 0.892 \\
\hline
\end{tabular}


Table 2. CI, HI and time of SIB plans

\begin{tabular}{|c|c|c|c|c|}
\hline \multirow{2}{*}{ T min } & \multicolumn{2}{|c|}{ PTV HR } & \multicolumn{2}{c|}{ PTV LR_nR } \\
\cline { 2 - 5 } & HI & CI & HI & CI \\
\hline 7.3 & 0.077 & 0.827 & 0.073 & 0.824 \\
\hline 7.5 & 0.056 & 0.734 & 0.063 & 0.754 \\
\hline 6.8 & 0.051 & 0.815 & 0.063 & 0.821 \\
\hline 6.6 & 0.078 & 0.9 & 0.081 & 0.808 \\
\hline 6.4 & 0.113 & 0.863 & 0.074 & 0.805 \\
\hline 6.8 & 0.065 & 0.738 & 0.073 & 0.852 \\
\hline 7 & 0.073 & 0.783 & 0.077 & 0.848 \\
\hline 6.4 & 0.056 & 0.682 & 0.075 & 0.851 \\
\hline
\end{tabular}

For both SEQ and SIB techniques, the organs at risk have received a dose that did not exceed the tolerance doses according to the QUANTEC [4], except plans, in which these structures had common points with PTV volume.

For SEQ plans critical organs may have common parts with PTV46 and PTV6o. Depending on that fact, different statistics were received for the same AORs (Table 3).

Table 3. Dose statistics for OARs for SEQ plans

\begin{tabular}{|l|c|c|c|}
\hline OARs & $\begin{array}{l}\text { Not included } \\
\text { in any PTV }\end{array}$ & $\begin{array}{l}\text { Particularly or } \\
\text { fully included } \\
\text { only in PTV46 }\end{array}$ & $\begin{array}{l}\text { Particularly or } \\
\text { fully included } \\
\text { in PTV46 and } \\
\text { PTV6o }\end{array}$ \\
\cline { 2 - 4 } & $\begin{array}{l}\text { Mean value of } \\
\text { all Dmax, Gy }\end{array}$ & $\begin{array}{l}\text { Mean value of } \\
\text { all Dmax, Gy }\end{array}$ & $\begin{array}{l}\text { Mean value of } \\
\text { all Dmax, Gy }\end{array}$ \\
\hline $\begin{array}{l}\text { Optic } \\
\text { nerve L }\end{array}$ & $\begin{array}{c}19.38 \\
(0.65-39.84)\end{array}$ & $\begin{array}{c}51.11 \\
(49.11-52.46)\end{array}$ & $\begin{array}{c}54.09 \\
(53.28-54.73)\end{array}$ \\
\hline $\begin{array}{l}\text { Optic } \\
\text { nerve R }\end{array}$ & $\begin{array}{c}19.08 \\
(0.66-41.52)\end{array}$ & $\begin{array}{c}54.26 \\
(45.43-51.77)\end{array}$ \\
\hline Chiasm & $\begin{array}{c}13.93 \\
(1.04-31.39)\end{array}$ & $\begin{array}{c}49.60 \\
(45.76-51.40)\end{array}$ & $\begin{array}{c}54.40 \\
(53.82-559\end{array}$ \\
\hline $\begin{array}{l}12.93 \\
\text { Brain } \\
\text { stem }\end{array}$ & $\begin{array}{c}52.66 \\
(5.67-20.19)\end{array}$ & $\begin{array}{c}58.33 \\
(54.33-56.35)\end{array}$ \\
\hline Lens L & $\begin{array}{c}5.11 \\
(0.42-9.80)\end{array}$ & - & - \\
\hline Lens R & $\begin{array}{c}5.10 \\
(0.40-9.8)\end{array}$ & - & - \\
\hline \hline & $\begin{array}{c}\text { Mean value } \\
\text { Dmean, Gy }\end{array}$ & - & - \\
\hline Eye L & $\begin{array}{c}6.74 \\
(0.43-13.05)\end{array}$ & - & - \\
\hline Eye R & $\begin{array}{c}10.80 \\
(0.40-21.20)\end{array}$ & - & - \\
\hline
\end{tabular}

For SIB plans critical organs dose is shown in Table 4 .

Table 4. Dose statistics for OARs for SIB plans

\begin{tabular}{|c|c|c|}
\hline \multirow[b]{2}{*}{ OARs } & \multicolumn{2}{|c|}{ Particularly or fully included in PTVLR } \\
\hline & $\begin{array}{l}\text { Mean value of all } \\
\text { Dmax, Gy }\end{array}$ & $\begin{array}{l}\text { Mean value of } \\
\text { all Dmax, } \\
\text { EQD2, Gy }\end{array}$ \\
\hline Optic nerve L & $\begin{array}{c}29.80 \\
(29.23-30.67) \\
\end{array}$ & $\begin{array}{c}35.6 \\
(34.6-37.3) \\
\end{array}$ \\
\hline Optic nerve R & $\begin{array}{c}29.65 \\
(28.67-30.29) \\
\end{array}$ & $\begin{array}{c}35 \cdot 5 \\
(33.7-36.5)\end{array}$ \\
\hline Chiasm & $\begin{array}{c}30.08 \\
(29.93-30.27) \\
\end{array}$ & $\begin{array}{c}36.0 \\
(35.8-36.5) \\
\end{array}$ \\
\hline Brain stem & $\begin{array}{c}30.68 \\
(30.22-32.87)\end{array}$ & $\begin{array}{c}37 \cdot 3 \\
(36.4-41.4) \\
\end{array}$ \\
\hline Lens L & $7.01(5.37-9.66)$ & - \\
\hline Lens R & $7.09(5.63-9.45)$ & - \\
\hline & Mean value Dmean, Gy & \\
\hline Eye L & $9.32(6.82-13.08)$ & - \\
\hline Eye R & $9.34(6.95-13.35)$ & - \\
\hline
\end{tabular}

Only brain stem was particularly or fully included in PTV40. Mean value of DmaxAv was 38.72 Gy (38.53 Gy $-38.92 \mathrm{~Gy})$ and EQD2 (a/b=3) was 53.2 Gy (52.7 Gy $-53.6 \mathrm{~Gy})$.

\section{CONCLUSIONS}

In case of using TomoTherapy planning system for creating therapeutic plans, the distribution of the dose in targets was characterized with high homogeneity and conformity; critical organs were protected as good as possible even in the cases where organs at risk were in close proximity to the target. The main feature of the TomoTherapy plans was a high gradient within the target dose of critical organs (even with using static jaws), and the ability to control the dose maximums in those areas, where a part or all critical organs had common points with the target volume, which makes it possible to provide good dose covering of PTV and to maximize the protection of organs at risk. To reach good results, additional third party contouring software was needed. Automated workflows for additional structure creation and TPS templates were implemented for reducing the time of preparing before starting the optimization of a plan.

To make treatment planning faster, more flexible and more interactive, VoLO technology, which combines the power of graphics processing units (GPUs) with a Non-Voxel Broad Beam (NVBB) algorithm is needed.

Using of Dynamic Jaws technology can reduce treatment time compared with the conventional 1.0-cm field width Fixed Jaws mode in SRT for brain metastasis. But no significant differences in dose distribution, clinical efficacy and toxicity were observed in study [5]. Thus, this technology is most useful in SRT.

\section{REFERENCES}

1. K. Tejinder et al., "Homogeneity Index: An objective tool for assessment of conformal radiation treatments," J. Med. Phys., vol. 37, no. 4, pp. 207-213, Oct-Dec. 2010.

DOI: 10.4103/0971-6203.103606

PMid: 23293452

PMCid: PMC3532749

2. I. Paddick, "A simple scoring ratio to index the conformity of Conformity index," J. Neurosurg., vol. 93, no. suppl. 3, pp. 219 - 222, Dec. 2000.

DOI: $10.3171 /$ jns.2000.93.supplement

PMid: 11143252

3. M.S. Arie van't Riet, A. C. Mak, M. A. Moerland, L. H. Elders, W. van der Zee, "A conformation number to quantify the degree of conformality in brachytherapy and external beam irradiation: application to the prostate," Int. J. Radiation Oncology Biol. Phys., vol. 37, no. 3, pp. 731-736, Feb. 1997.

DOI: $10.1016 /$ So360-3016(96)oo601-3

PMid: 9112473

4. L. B. Marks, E. D. Yorke et al., "Use of normal tissue complication probability models in the clinic," Int. $J$. Radiat. Oncol. Biol. Phys., vol. 76, no. 3, pp. 10-19, Mar. 2010.

DOI: 10.1016/j.ijrobp.2009.07.1754

PMid: 20171502

PMCid: PMC4041542 
5. T. Murai, A. Hayashi, Y. Manabe et al., "Efficacy of stereotactic radiotherapy for brain metastases using dynamic jaws technology in the helical tomotherapy system," Br. J. Radiol., vol. 89, no. 1066, Oct. 2016.

DOI: 10.1259/bjr.20160374

PMid: 27556639

PMCid: $\mathrm{PMC}_{5124807}$ 